\title{
Protective Effect of Catalpol on Myocardium in Rats with Isoprenaline-Induced Myocardial Infarcts via Angiogenesis through Endothelial Progenitor Cells and Notch1 Signaling Pathway
}

\author{
Jing Zeng ${ }^{1}$, Feng Huang ${ }^{1}$, Yuangqing $\mathrm{Tu}^{1}$, Saichun $\mathrm{Wu}^{1}$, Manping $\mathrm{Li}^{1}$, Xiaoyun Tong ${ }^{2 *}$ \\ ${ }^{1}$ College of Pharmacy, Jinan University, Guangzhou, China; ${ }^{2}$ Teaching \& Research Section of Internal Medicine of Traditional Chi- \\ nese Medicine, College of Clinical Medicine, Yunnan University of TCM, Kuming, China. \\ Email: lanmaomaono1@gmail.com, txytong@hotmail.com
}

Received September $20^{\text {th }}, 2013$; revised October $21^{\text {st }}, 2013$; accepted October $28^{\text {th }}, 2013$

Copyright (C) 2013 Jing Zeng et al. This is an open access article distributed under the Creative Commons Attribution License, which permits unrestricted use, distribution, and reproduction in any medium, provided the original work is properly cited.

\begin{abstract}
Protective effect of catalpol on myocardium was studied in relation to endothelial progenitor cells, Notch1 signaling pathway and angiogenesis in rats with isoprenaline (INN)-induced acute myocardial infarcts. To analyze the pathological status and impact of catalpol on the rats, 3 weeks after intragastric gavage, the animals were verified for myocardial infarcts with electrocardiogram and measured for enzyme activity of lactate dehydrogenase (LDH), malondialdehyde (MDA), creatine kinase (CK) and superoxide dismutase (SOD) in myocardium, and further analyzed using HE and TTC staining, as well as visual examination of infarct area. Flow cytometry study of endothelial progenitor cells (EPCs) indicated that the EPCs were mobilized during infarction. The roles of Notch1 signaling pathway in angiogenesis of the infracted animals were studied using immunohistochemistry analysis of RBPj $\kappa$ and Western blot analysis of Notch1 and Jagged1. Our results obtained from the rats treated with catalpol, positive drug and control showed that catalpol could protect rats from infarction probably by mobilization of EPCs and activation of Notch1 signaling pathway.
\end{abstract}

Keywords: Myocardial Infarction; Endothelial Progenitor Cell; Notch1 Signaling Pathway; Angiogenesis; Catalpol

\section{Introduction}

Acute myocardial infarction caused by ischemic cardiomyopathy is one of the major human diseases and has the highest mortality and morbidity among all diseases. Prevention and control of the disease is number one healthy issue globally. Many options have become available to treat the disease, such as thrombolytic therapy and percutaneous coronary intervention. However, not all patients are suitable for these therapies due to non-compliant and ineffectiveness in restoration of blood supply. Therefore, search for new options to treat myocardial infarction has been a hotspot in cardiovascular disease research. Myocardial infarction decreases or blocks the blood supply of coronary artery due to the damage in coronary artery, resulting in severe and long-lasting ischemia of myocardial muscle, and eventually ischemic necrosis of the affected muscle. On the other hand, an-

${ }^{*}$ Corresponding author. giogenesis can improve the blood circulation in coronary collateral artery and restore the supply of blood to the ischemic myocardium, reducing the death of myocardium cells. EPCs exist in bone marrow and peripheral blood, which could differentiate into endothelial cells [1]. Since the isolation of EPCs from peripheral blood in 1997 by Ashahara, which could differentiate into endothelial cells and are involved in angiogenesis, the roles of EPCs in angiogenesis or vasculogenesis have attracted considerable attention. Many studies have demonstrated that EPCs play roles in the postnatal neovascularization and restoration of injured blood vessel endothelium [2]. When induced by cytokines released by ischemic tissue, EPCs can be mobilized from bone marrow to peripheral blood to circulate, migrate, home to the injured area, where they proliferate and differentiate into endothelial cells to involve in blood restoration and neovascularization [3]. Notch signaling is an important signal pathway that extensively exists in vertebrates and invertebrates [4]. 
It has been shown that the differentiation of endothelial cells is regulated by the pathway. In addition, it also participates in the vascularization of adult. Therefore, a better understanding of relationship between Notch signal pathway and EPCs would be scientifically and clinically important to improve neovascularization and restoration of ischemic myocardium. Notch1 has been reported to play a key role in angiogenesis [5]. EPCs were found to decline dramatically in mice that had been knocked out for Jagged1, the ligand of Notch1 receptor [6]. Therefore, Jagged 1 is believed to have an important role in determining the number of EPCs and their mobilization into blood for restoration of injured blood vessels [7]. Clinical data have shown that prescriptions that nourish kidney and activate blood are effective in treatment of acute myocardial infarction [8]. Previous animal studies have demonstrated that the prescriptions improved the release of bone marrow stem cells into peripheral blood, leading to increase in number of CD34+ cells and restoration of the injured muscles [9]. Catalpol, an iridoid glucoside separated from the roots of Rehmannia glutinosa, is the major active integrant in a Chinese medical prescription that nourishes kidney and activates blood. It has been shown to be neuroprotective in transient global ischemia in gerbils [10]. However, little is known about the role of catalpol in EPCs and neovascularization in myocardium. In this study, we investigated the repair process of blood vessel by catalpol and analyzed if Notch1 signal pathway is involved in promoting the release of EPCs into peripheral blood and restoring neovascularization in damaged myocardium.

\section{Materials and Methods}

\subsection{Animals}

42 healthy adult SD rats, male, weighting $200 \pm 20$ g, were purchased from Guangdong Experimental Animal Center (quality assurance permit no. SCXK 2008-0002).

\subsection{Reagents and Instruments}

Catalpol was obtained from Medicine Inspecting Institute of Guangdong province. The purity was confirmed by HPLC to be $98 \%$. Isoprenaline (INN) (lot no. CBC7466) was purchased from Sigma. MDA detection kit (lot no. 20121010), LDH detection kit (lot no. 20121010), SOD detection kit (lot no. 20121011) and CK detection kit (lot no. 20121010) were purchased from Nanjing Jiancheng Bioengineering Institute. Dimethyl benzene (lot no. 20120120), hematoxylin (lot no. 07H08A05) and neutral balsam (lot no. 20111215) were purchased from Guangzhou Weijia technology company. PerCP-Cy5.5-CD34 and rat-anti Jaggedl antibody (sc-6011) were obtained from Santa Cruz Biotechnology, INC. FITC conjugated rabbit anti-VEGFR2/VEGFR2 antibody, rabbit anti-
CD133 antigen/PE and rat-anti Notch1 antibody were from Cell Signaling Technology. BCA protein quantification kit and RIPA lysis buffer (lot no. P0012 and P0013B) were purchased from Biyuntian Biotechnology Institute. APS, Acr-Bic and Tween 20 were from Amersco. Tris-base, SDS and glycine were purchased from Guangzhou Pubo Instrument Company. PVDF was from Osmonics (USA). Protein marker (lot no. 00061924) was from MBI Fermentas (Canada). Chemiluminescence solution (ECL) was from PBP1001 (USA) (lot no. 26). Multifunctional full wavelength micro plate reader (Synergy 2) was purchased from Biotek (USA), inverted microscope (TS-100F) was from Nilsson (Japan).

\section{Experimental Contents}

\subsection{Animal Model}

All experimental procedures were conducted in compliance with institutional guidelines for the care and use of laboratory animals in SPF laboratory, experimental animal research center of Jinan University, Guangzhou, China.

The rats were randomly divided into the following six groups with 7 animals each: control, model, positive, low $(10 \mathrm{mg} / \mathrm{kg})$, middle $(20 \mathrm{mg} / \mathrm{kg})$ and high dose $(40 \mathrm{mg} / \mathrm{kg})$ catalpol. For animals used in control and model groups, they were gavaged for three weeks with physiological saline and were injected subcutaneously with physiological saline or INN $(10 \mathrm{mg} / \mathrm{kg})$ on 19th, 20th and 21th day to induce acute myocardial infarction. Rats in positive group were gavaged with Simvastatin dissolved in physiological saline $(1.5 \mathrm{mg} / \mathrm{kg}, 1 \mathrm{~mL} / 100 \mathrm{~g})$ for 3 weeks, and then injected subcutaneously with INN on 19th, 20th and 21 th day $(10 \mathrm{mg} / \mathrm{kg})$ to induce acute myocardial infarction. For catalpol group rats were injected subcutaneously with INN on 19th, 20th and 21th day $(10 \mathrm{mg} / \mathrm{kg})$ after 3 week gavage with catalpol $(1 \mathrm{~mL} / 100 \mathrm{~g})$.

\subsection{Electrocardiogram}

Within 4 hours after the last INN injection, the animals were anesthetized by intraperitoneally injection with $10 \%$ chloral hydrate $(0.35 \mathrm{~mL} / 100 \mathrm{~g}) .5 \mathrm{~min}$ later, electrocardiograms of the rats in all groups were simultaneously made with electrocardiograph VII (paper speed $50 \mathrm{~mm} / \mathrm{s}$ ) to record the change in EGG-ST segment and the pathological T wave.

\subsection{Enzyme Assays}

22 days after the gavage, rats were anesthetized and blood collected from abdominal aorta in two tubes for each rat, one containing coagulant and the other containing EDTA as anticoagulant. The coagulant tubes were left on rack for $2 \mathrm{~h}$ and then centrifuged at $4^{\circ} \mathrm{C}$ for 20 
$\min$ at $3500 \mathrm{rpm}$. The supernatants were collected to determine the contents of $\mathrm{LDH}, \mathrm{SOD}, \mathrm{CK}$ and MDA. The blood in the anticoagulant tubes was used immediately for subsequent EPC flow cytometry analysis.

\subsection{EPC Flow Cytometry}

For each assay, a sample and blank tube was used. In the blank tubes, only PerCP-Cy5.5-CD34 antibody was added for gating, but not CD133+ and KDR antibody. In the sample tubes, $5 \mu 1$ each of the three antibodies was added and mixed with $100 \mu \mathrm{l}$ blood, incubated at the dark for $20 \mathrm{~min}$, lysed for at least $5 \mathrm{~min}$ by adding $1 \mathrm{ml}$ of erythrocyte lysis buffer till the blood solution was completely transparent. The lysed blood samples were pelleted at $1000 \mathrm{rpm}$ for $5 \mathrm{~min}$, washed with PBS, votexed and centrifuged again. The pellets were resuspended in $200 \mu \mathrm{l}$ PBS and used for flow cytometry assay.

\subsection{TTC Staining}

The rats were injected $20 \mathrm{ml}$ of $1 \%$ TTC via abdominal aorta following collecting the blood as described in previous section. After staining for $10 \mathrm{~min}$, the chests were opened and hearts taken to dissect for photograph. The sections were fixed in formalin and photographed again for better contrast.

\subsection{HE Staining}

Paraffin sections were dewaxed, stained hematoxylin solution for $5 \mathrm{~min}$, washed $1 \mathrm{~min}$ in running tap water. They were then hydrated for $30 \mathrm{~s}$ in $75 \%$ hydrochloric acid-alcohol and washed 2 min in water. After treated with ammonia for $30 \mathrm{~s}$ and washed with water for $1-2$ min, the slides were dehydrated through an alcohol gradient, sealed with neutral balsam after clarified with dimethyl benzene and viewed under a microscope for pathological changes.

\subsection{Immunohistochemistry}

Paraffin sections were baked at $60^{\circ} \mathrm{C}$, dewaxed and hydrated through dimethyl benzene and ethanol serials. The slides were finally washed with distilled water and incubated in sodium citrate buffer in a microwave oven for 5 min at high power to restore antigen. After cooling down at room temperature, they were washed two times for 5 min each with TBS and incubated in $3 \% \mathrm{H}_{2} \mathrm{O}_{2}$ for 30 min. The slides were then washed two time with TBS for 5 min each, and blocked with $10 \%$ goat serum for $30 \mathrm{~min}$, and reacted with 1:20 diluted first antibody (anti CD34 antibody). After incubation at $37^{\circ} \mathrm{C}$ for $60 \mathrm{~min}$ or at $4^{\circ} \mathrm{C}$ overnight, the samples were washed two times with TBST for $5 \mathrm{~min}$ each, and stained with DAB for $10 \mathrm{~min}$.
After the staining, the slides were washed with running tap water and stained with hematoxylin for 60 seconds, washed 7 to 8 times and under running water for $3 \mathrm{~min}$. The slides were dehydrated through an ethanol and dimethyl benzene serial, and sealed with neutral balsam and observed under a microscope.

\subsection{Western Blot Analysis}

$100 \mathrm{mg}$ of $-80^{\circ} \mathrm{C}$ frozen stored myocardial tissue was homogenated in $400 \mu \mathrm{L}$ RIPA lysis buffer in an ice bath for $2 \mathrm{~min}$, and incubated in the ice bath for $15 \mathrm{~min}$ before centrifugation at $4^{\circ} \mathrm{C}$ for $10 \mathrm{~min}$. The supernatants were transferred to $1.5 \mathrm{~mL}$ Eppendorf tubes for protein quantification according to the BCA kit manual. $45 \mu \mathrm{g}$ of protein was taken from each sample for electrophoresis and transferred to the PVDF membranes after the electrophoresis. The membranes were blocked with TBST (10 $\mathrm{mmol} / \mathrm{L}$ Tris- $\mathrm{HCl}, \mathrm{pH} 7.5,150 \mathrm{mmol} / \mathrm{L} \mathrm{NaCl}, 1 \%$ Tween-20) containing 5\% skim milk powder incubated and reacted with Notch1 antibody (1:1000 dilution) and Jagged 1 antibody (1:1000 dilution) overnight at $4^{\circ} \mathrm{C}$. After washed with TBST, the membranes were incubated with secondary antibody (1:2000 dilution) for $1 \mathrm{~h}$, washed and reacted with ECL for $1 \mathrm{~min}$. The images were exposed and captured on X-ray films.

\section{Results}

\subsection{Electrocardiogram}

As shown in Figure 1, compared with control group, rats in model group had an abnormal raise in S-T segment. Meanwhile, Simvatastin and catalpol at doses used could reduce the elevation.

\subsection{Enzyme Assays}

Results showed that the activity of LDH (Figure 2) in model group was $17111.1 \mathrm{U} / \mathrm{L}$, significantly higher than that of control $(6105.5 \pm 542.6 \mathrm{U} / \mathrm{L}, \mathrm{p}<0.01)$. This confirmed that the rats in the model group were infracted. After given catalpol at the doses used, LDH activities were reduced to $7343.1 \pm 2110.1,5352.9 \pm 3070.3$, and $3705.9 \pm 624.5 \mathrm{U} / \mathrm{L}$ at low, middle and high dose, respectively.

In the model group, the SOD level (Figure 3) was lower than that in control $(65.0 \pm 6.6$ vs. $7.1 \pm 3.3 \mathrm{U} / \mathrm{mL})$, indicating that the antioxidation ability in the acutely infracted muscle was reduced. After given catalpol, SOD activity was increased slightly to $70.1 \pm 8.1,76.7 \pm 4.6$ and $81.1 \pm 11.2$ at the three doses levels, respectively.

As shown in Table 1, the CK level of rats (Figure 4) in model group was significantly higher than that of control $(12.2 \pm 2.0$ vs. $7.1 \pm 3.3 \mathrm{U} / \mathrm{mL}, \mathrm{p}<0.01)$, indicating 


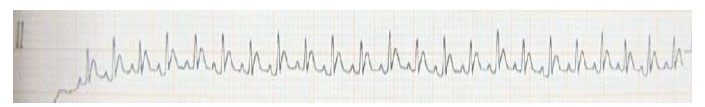

(a)

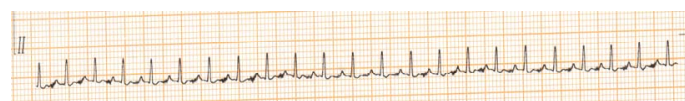

(c)

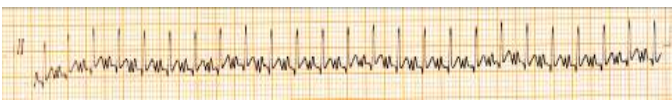

(e)

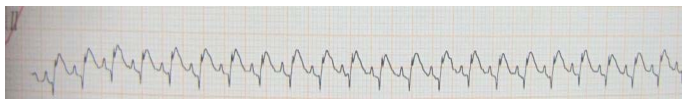

(b)

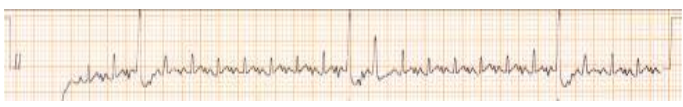

(d)

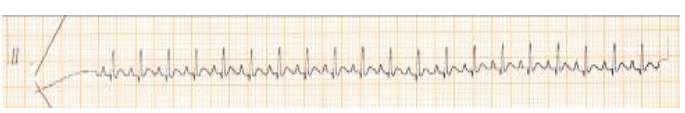

(f)

Figure 1. Representative electrocardiograms of rats with INN-induced infraction. (a) Control; (b) INN; (c) Simvastatin; (d) Catalpol (10 mg/kg); (e) Catalpol (20 mg/kg); (f) Catalpol (40 mg/kg).

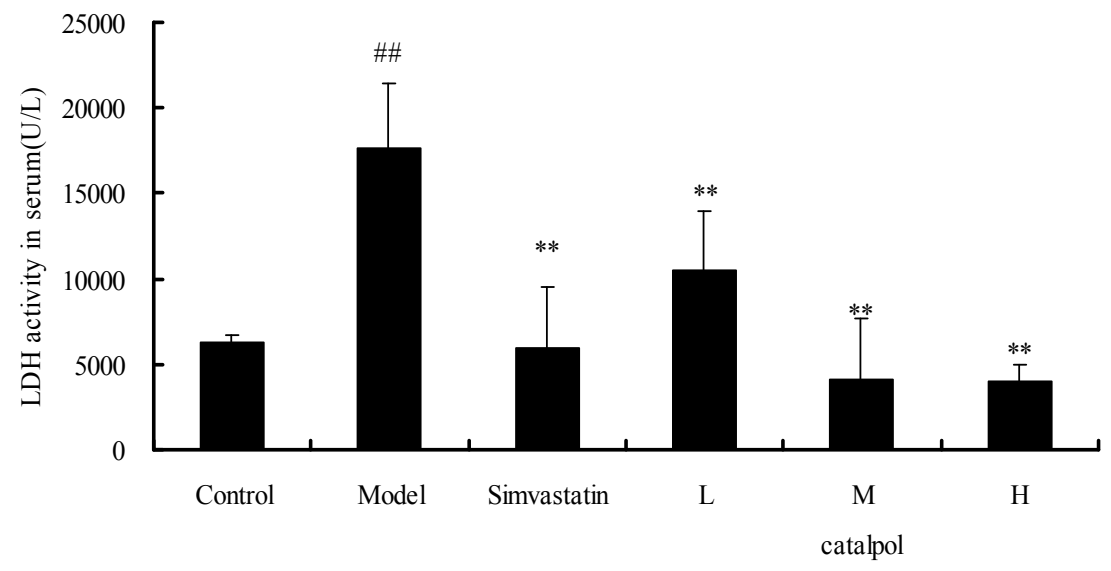

Figure 2. Effect of catalpol on serum LDH activity in rats with myocardium infarction induced by INN $\left({ }^{\#} p<0.05\right.$, ${ }^{\#} p<0.01$ vs control; ${ }^{*} \mathrm{p}<0.05,{ }^{* *} \mathrm{p}<0.01$ vs model).

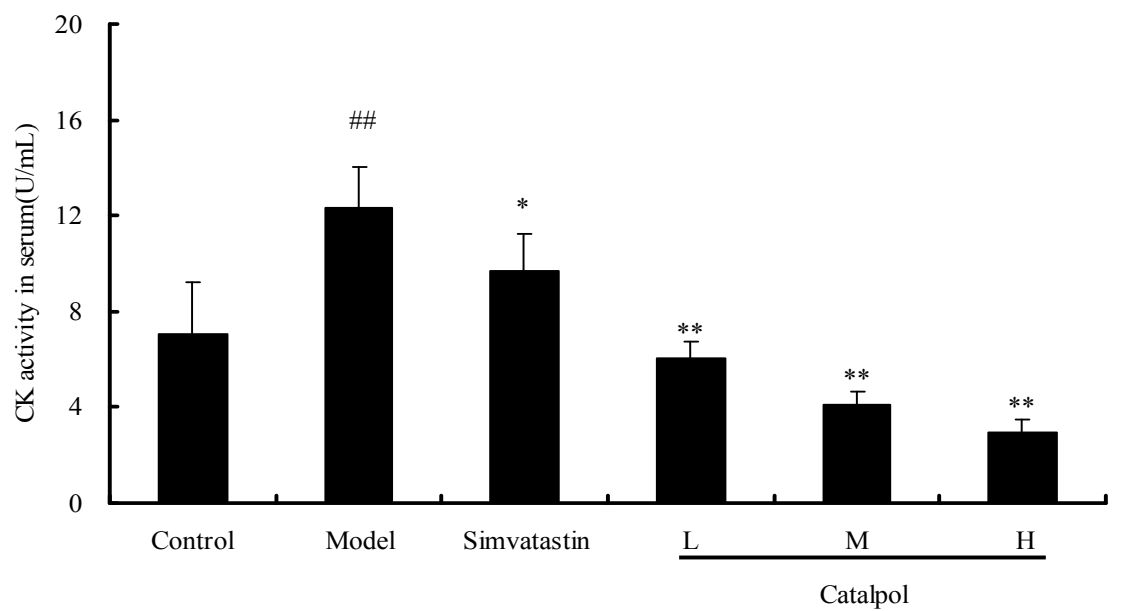

Figure 3. Effect of catalpol on serum SOD activity of rats with myocardium infarction induced by INN $\left({ }^{\#} p<0.05\right.$, \# $p<0.01$ vs control; ${ }^{*} \mathbf{p}<0.05,{ }^{* *} \mathbf{p}<0.01$ vs model).

that injury of the myocardial tissue had led to increased CK activity. Catalpol treatments were found to reduce the activities to $5.1 \pm 0.7,3.6 \pm 0.2$ and $2.8 \pm 0.8 \mathrm{U} / \mathrm{mL}$ at the three doses, respectively, in a dose-dependent manner.
Data in Table 1 shows that MDA content in model group was significantly higher than in control $(p<0.01)$, indicating that there was an increase in serum MDA in the infracted rats. After given catalpol, MDA levels (Figure 5) were decreased to $4.6 \pm 1.2,4.5 \pm 0.6$ and $2.5 \pm$ 

Angiogenesis through Endothelial Progenitor Cells and Notch1 Signaling Pathway

Table 1. Effect of catalpol on LDH, SOD, CK and MDA activity of rats with INN-induced myocardium infarction.

\begin{tabular}{cccccc}
\hline Group & $\mathrm{n}$ & $\mathrm{LDH} \mathrm{U} / \mathrm{L}$ & SOD U/mL & CK U/mL & $\mathrm{MDA} \mathrm{nmol} / \mathrm{mL}$ \\
\hline Control & 6 & $6246.4 \pm 464.9$ & $78.0 \pm 10.1$ & $7.0 \pm 2.2$ & $1.7 \pm 0.3$ \\
Model & 6 & $17602.8 \pm 3846.5^{\# \#}$ & $62.1 \pm 14.7^{\text {\#\# }}$ & $12.3 \pm 1.7^{\# \#}$ & $4.2 \pm 0.9^{\# \#}$ \\
Simvastatin & 6 & $5904.8 \pm 3565.5^{* *}$ & $84.2 \pm 5.8^{* *}$ & $9.7 \pm 1.5^{*}$ & $2.0 \pm 0.5^{* *}$ \\
CAT 10 mg/kg & 6 & $10534.3 \pm 3473.1^{* *}$ & $68.6 \pm 6.5$ & $6.0 \pm 0.8^{* *}$ & $4.4 \pm 1.0^{*}$ \\
CAT 20 mg/kg & 6 & $4152.0 \pm 3545.5^{* *}$ & $77.2 \pm 4.0^{*}$ & $4.1 \pm 0.5^{* *}$ & $2.9 \pm 0.4^{* *}$ \\
CAT 40 mg/kg & 6 & $3983.8 \pm 949.1^{* *}$ & $81.1 \pm 8.4^{*}$ & $3.0 \pm 0.5^{* *}$ & $2.9 \pm 0.6^{* *}$ \\
\hline
\end{tabular}

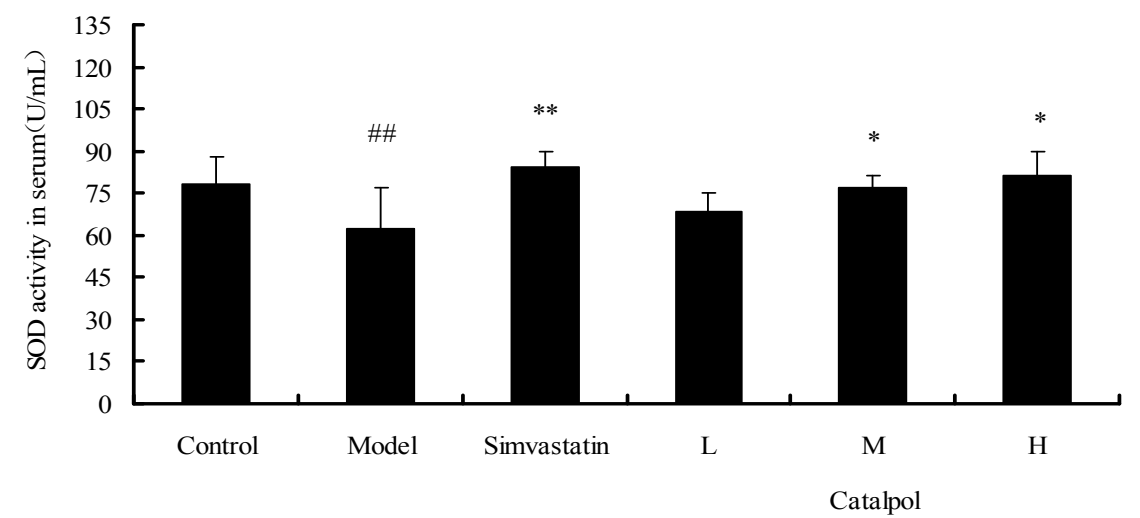

Figure 4. Effect of catalpol on serum CK activity of rats with myocardium infarction induced by INN $\left({ }^{\# p}<0.05\right.$, ${ }^{\# \#}<0.01$ vs control; ${ }^{*} \mathrm{p}<0.05,{ }^{* *} \mathrm{p}<0.01$ vs model).

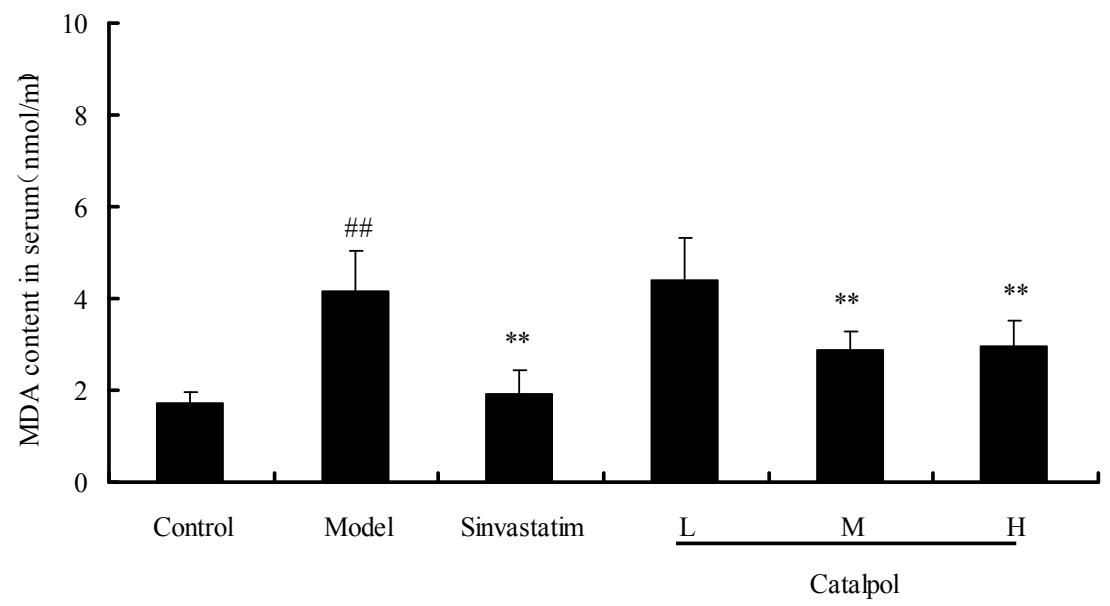

Figure 5. Effect of catalpol on the serum content of MDA in of rats with myocardium infarction induced by INN ( ${ }^{*} p<0.05$, ${ }^{\#} \mathrm{p}<\mathbf{0 . 0 1}$ vs control; ${ }^{*} \mathrm{p}<\mathbf{0 . 0 5},{ }^{* *} \mathrm{p}<\mathbf{0 . 0 1}$ vs model).

$1.9 \mathrm{nmol} / \mathrm{mL}$ at the three dose levels, respectively.

\subsection{EPC Flow Cytometry}

In this study, gating was made for CD34. The CD34 positive events were counted for CD133+/VEGFR2+ double positive cells and expressed as percentage. As shown in Figure 6, the peripheral blood EPC counts for CD34+/CD133+/VEGFR2+ events were higher in model than in control. After catalpol treatments, the counts increased slightly at all dose levels to $0.51 \%, 0.97 \%$ and
$3.22 \%$, respectively.

\subsection{TTC Staining}

As shown in Figure 7, model rats had larger white areas than control after TTC staining. Rats receiving Simvastatin and catalpol had smaller white areas, indicating that the infract areas were reduced.

\subsection{HE Staining}

In normal rats, the myocardial cells were regularly ar- 


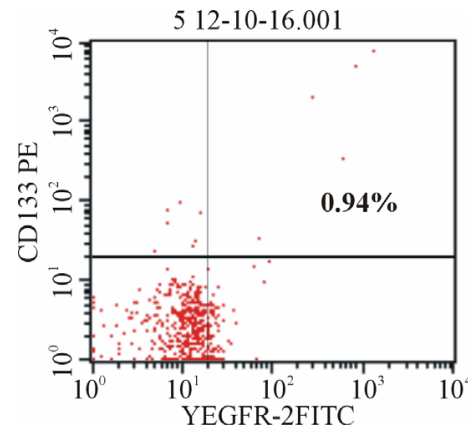

(a)

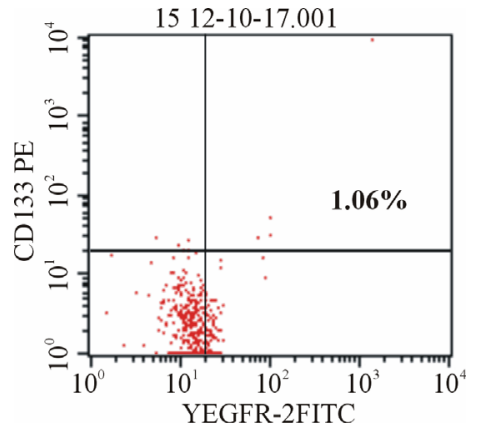

(d)

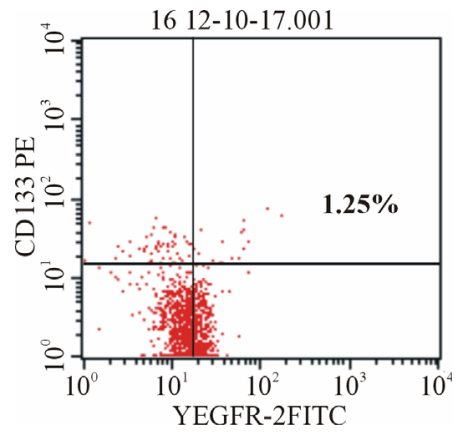

(b)

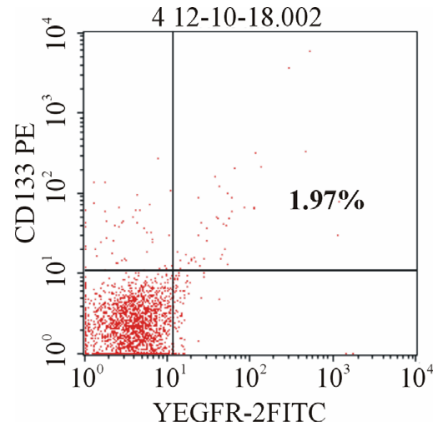

(e)

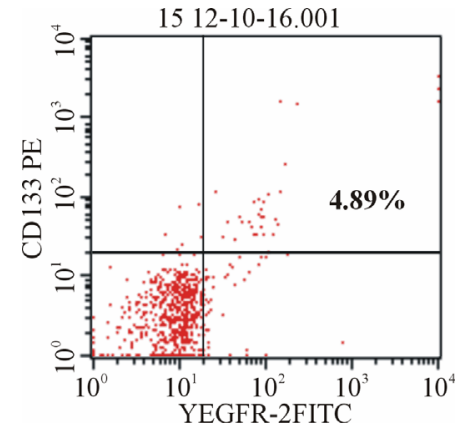

(c)

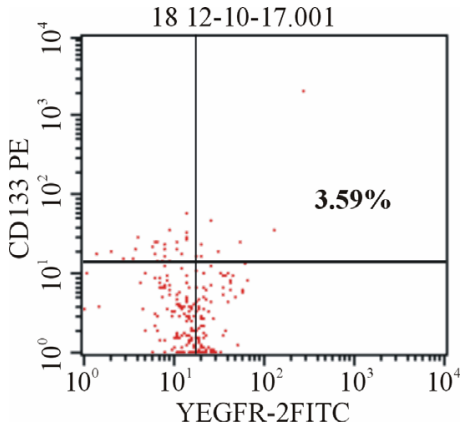

(f)

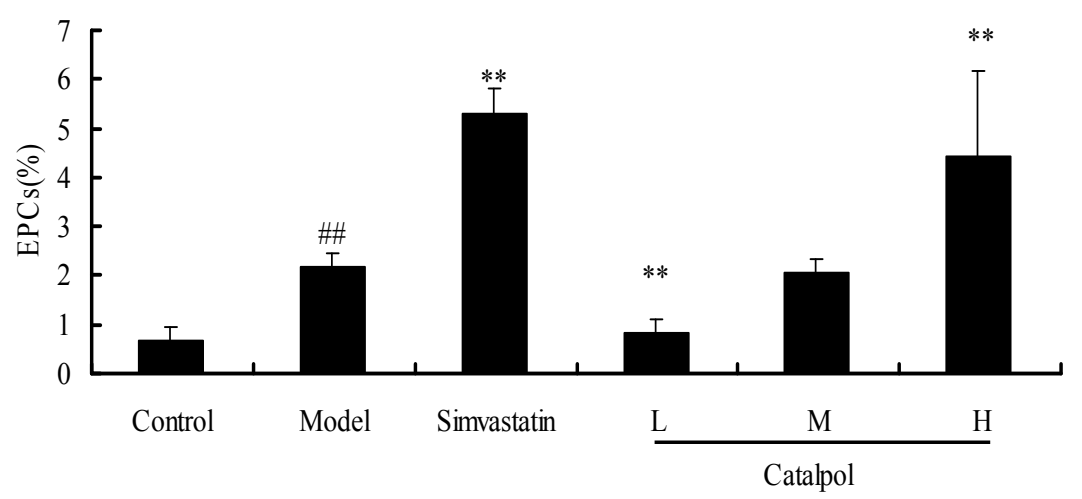

Figure 6. Effect of catalpol on the number of EPCs in peripheral blood of rats with myocardial infarction induced by INN. (a) control; (b) ISO; (c) Simvastatin; (d) Catalpol (10 mg/kg); (e) Catalpol (20 mg/kg); (f) Catalpol (40 mg/kg), $\left(^{\#} \mathrm{p}<0.05\right.$, ${ }^{\#}$ p $<$ 0.01 vs control; ${ }^{*} \mathrm{p}<0.05,{ }^{* *} \mathrm{p}<0.01$ vs model).

ranged with intact morphology and unchanged nuclei. The staining was uniform with clearly striated muscle fibers and without swollen and necrosed cells (Figure 8(a)). In the infracted rats, arrangements of myocardial cells were disrupted, cardiac muscle fiber swollen with increased cytoplasm acidophilia. The nuclei were seen shrunk or broken, and invasioned by inflammatory cells (Figure 8(b)). Myocardial striated muscle disappeared and nucleus become dissolved, resulting in necrosed myocardial muscle. In comparison with catalpol, Simvastatin treated rats had smaller amount of inflammatory cell infiltration and leaking of red blood cells (Figure 8(c)). At low and middle catalpol doses, there were some inflammatory cell infiltration with partially dissolved myocardial fibers and increased acidophilia (Figures 8(d) and (e)). At high catalpol dose, myocardial cells were mostly intact with more regularly arranged muscle fibers and less inflammatory exudate (Figure 8(f)).

\subsection{Immunohistochemistry}

Immunohistochemistry study showed that there were slight brown staining in rats in model but not in control groups, indicating that $\mathrm{RBPj} \kappa$ were expressed slightly higher in the myocardial muscle of infracted rats than in control (Figures 9(a) and (b)). Simvastatin treatment resulted in deeper brown staining (Figure 9(c)). Increased staining was seen at middle and high dose of catalpol (Figures 9(d)-(f)) but not at low dose, although the staining was not as obvious as in Simvastatin-treated rats. 


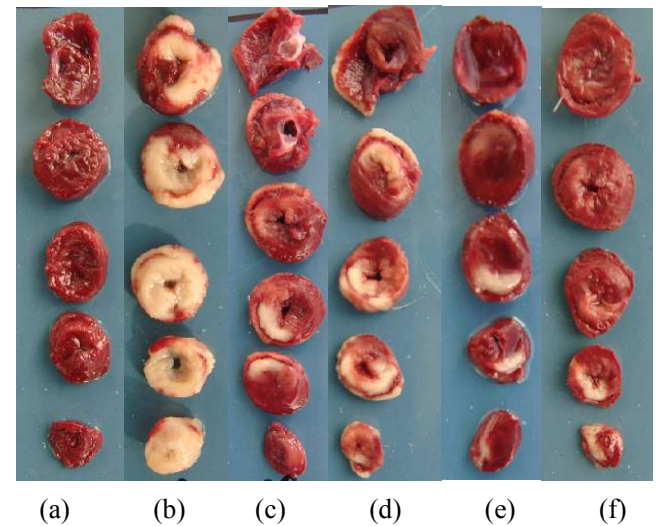

Figure 7. TTC staining of myocardium tissues from rats with INN-induced infraction. (a) Control; (b) INN; (c) Simvatastin; (d) Catalpol (10 mg/kg); (e) Catalpol (20 mg/kg); (f) Catalpol (40 mg/kg).

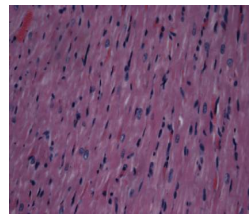

(a)

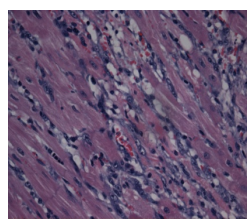

(d)

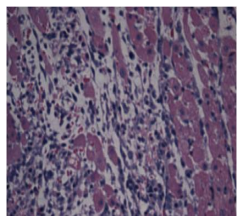

(b)

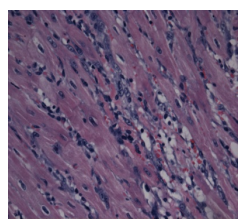

(e)

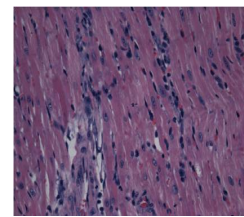

(c)

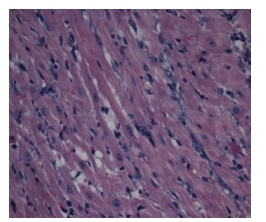

(f)
Figure 8. Effect of catalpol on tissue structure of rats with INN-induced myocardial infraction (H\&E, 200×). (a) Control; (b) INN; (c) Simvastatin; (d) Catalpol (10 mg/kg); (e) Catalpol (20 mg/kg); (f) Catalpol (40 mg/kg).

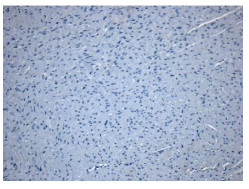

(a)

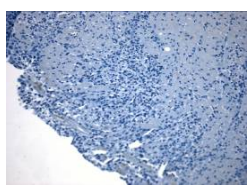

(d)

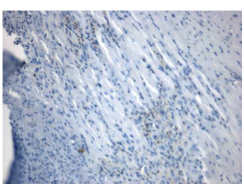

(b)

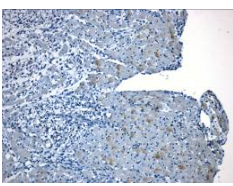

(e)

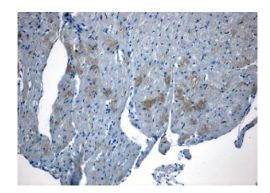

(c)

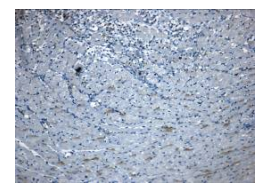

(f)
Figure 9. Effect of catalpol on RBPj $\boldsymbol{c}$ expression in cardiac tissue of rats with myocardial infarction induced by INN. (a) Control; (b) INN; (c) Simvastatin; (d) Catalpol (10 mg/kg); (e) Catalpol (20 mg/kg); (f) Catalpol (40 mg/kg).

\subsection{Western Blot Analysis}

To investigate the molecular mechanism of catalpol-in- duced mobilization of bone marrow-derived EPCs to peripheral blood in the infracted rats, we measured the expression of Notch1 and Jagged1 in the Notch signaling pathway in the myocardial tissues using Western blot analysis. As shown in Figure 10, Notch1 was found downregulated in the infracted rats, and it increased slightly after given catalpol. Jagged 1 was also reduced remarkably in the infracted rats and increased after catalpol treatments at all dose levels used. The increase was found to be dose-dependent. These findings indicated that changes in expression of Notch1 and Jagged1 were consistent each other.

\section{Discussion}

In this study, we injected INN subcutaneously to prepare the infract rat model. This is a widely used method. Myocardium infarction is a common and severe disease clinically. One of the most important methods to examine the myocardial injury is thorough the detection of biomarkers in blood [11,12]. In normal condition, LDH, an important enzyme involved in energy metabolism, exists widely in cardiac tissues. When the tissues are injured, the enzyme is released into blood, resulting in high serum LDH level. SOD plays important role in balancing the oxidation and antioxidation activities, and is a major oxygen free radical scavenger. The final product of lipid oxidation is MDA, which is an indirect indictor of cell injury, and therefore can be used to measure the damage in the cardiac tissues. Results from our study indicated that in the infracted rats, serum activities of LDH and CK increased significantly, while those of SOD reduced significantly. After feeding with medium or high dose of the prescription, the activities and levels of LDH, CK, SOD and MDA were all returned to normal, indicating that catalpol was able to scavenge oxygen free radicals, reduce the production of oxidized products from lipids and the damage in cardiac tissues. TTC staining can visualize the infract areas. Using TTC staining, we found that catalpol effectively reduced the infract areas and improve the pathological status of the infracted tissues. We also

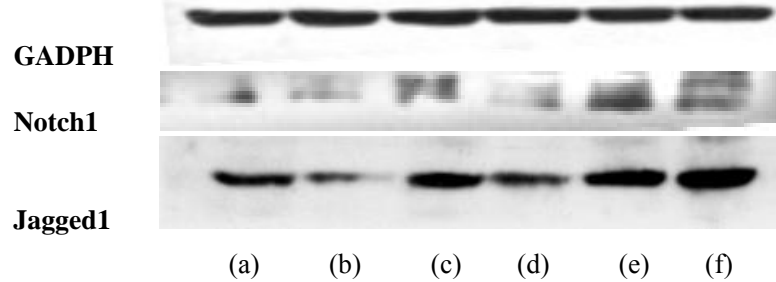

Figure 10. Effects of catalpol on the expression of Notch1 and Jagged1 in cardiac tissue of rats with myocardial infarction induced by INN. (a) Control; (b) INN; (c) Simvastatin; (d) Catalpol (10 mg/kg); (e) Catalpol (20 mg/kg); (f) Catalpol (40 mg/kg). 
showed that the size of infracting areas observed by TTC was in line with the data from the enzyme and biomarker assays. These results all showed that catalpol is an effective protective agent to myocardial ischemia.

EPCs are the endothelial progenitor cells. They are involved in embryonic vasculogenesis, angiogenesis after birth and repair of endothelial injury in blood vessels $[13,14]$. Studies have shown that EPCs in bone morrow trend to home to ischemic tissues. Once arriving in the ischemic tissues, they differentiate into myocardial cells and endothelial cells, to repair the impaired tissues. Normally, EPCs account for $0.1 \%$ of the peripheral blood. When ischemia occurs, bone morrow-derived EPCs are mobilized to enter peripheral blood at an amount that is not sufficient for the repair. Therefore, promotion of angiogenesis in myocardium tissue through clinical treatments is currently the hotspots of research and practice in treatment of myocardial ischemia. How to increase the proliferation and differentiation of EPCs is a new direction in treatment of coronary heart diseases. Clinically, ischemic diseases, particularly coronary heart diseases, are always associated with one or more risk factors for cardiovascular system. The risks are negatively related to the number of EPCs, which are considered as prognosis indicator for coronary heart diseases [15]. There are a number of methods to determine the amounts of EPCs. In most studies, CD34+, VEGFR-2+, and CD133+ are three mostly frequently used indicators. In this study, we investigated the CD34+, VEGFR-2+, and CD133+ events in peripheral blood, and found that EPCs were mobilized to peripheral blood in the infracted rats with or without drug treatment. These findings confirmed that EPCs are released from bone marrow to peripheral blood when the rats are stressed with dramatic shocks such as ischemia.

Notch signal pathway is first discovered in Drosophila, and made up of receptors (Notch1, Notch 2, Notch 3, and Notch 4), ligands (Jagged1, Jagged 2, Dll-1, Dll-3 and Dll-4) and DNA binding protein CSL. It has been shown that the differentiation of endothelial cells is mainly regulated via Notch signal pathway, and the endothelial cells have shown the potential to differentiate into artery and vein before blood perfusion [16]. Notch/Jagged1 is a newly discovered important angiogenesis factor. Western blot analysis indicated that the expression of Notch1 receptor in the myocardium was reduced remarkably in the infracted rats and in the normal rats [17]. Feeding of the rats with the drugs increased the expression, in a dose dependent way in case of catalpol, where high and medium doses were better than low dose. Therefore, we speculate that catalpol may activate Notch signal pathway to promote the differentiation and proliferation of endothelial cells in the infracted rats, and to improve the oxygen supply to ischemic and injured myocardial tis- sues, resulting in protection to myocardium and reduced infracted area.

\section{Acknowledgements}

This research was supported by National Natural Science Foundation of China (No. 81060295).

\section{REFERENCES}

[1] T. Asahara, T. Murohara, A. Sullivan, et al., "Isolation of Putative Progenitor Endothelial Cells for Angiogenesis," Science, Vol. 275, No. 5302, 1997, pp. 964-966. http://dx.doi.org/10.1126/science.275.5302.964

[2] T. Asahara, T. Takahashi, H. Masuda, et al., "VEGF Contributes to Postnatal Neovascularization by Mobilizing Bone Marrow-Derived Endothelial Progenitor Cells," The EMBO Journal, Vol. 18, No. 14, 1999, pp. 3964-3972. http://dx.doi.org/10.1093/emboj/18.14.3964

[3] T. Takahashi, C. Kalka, H. Masuda, et al., "Ischemia-and Cytokine-Induced Mobilization of Bone Marrow-Derived Endothelial Progenitor Cells for Neovascularization," $\mathrm{Na}$ ture Medicine, Vol. 5, No. 4, 1999, pp. 434-438. http://dx.doi.org/10.1038/7434

[4] T. Gridley, "Notch Signaling in Vertebrate Development and Disease," Molecular and Cellular Neuroscience, Vol. 9, No. 2, 1997, pp. 103-108.

http://dx.doi.org/10.1006/mone.1997.0610

[5] L. T. Krebs, Y. Xue, C. R. Norton, et al., "Notch Signaling Is Essential for Vascular Morphogenesis in Mice," Genes \& Development, Vol. 14, No. 11, 2000, pp. 13431352.

[6] Y. Xue, X. Gao, C. E. Lindsell, et al., "Embryonic Lethality and Vascular Defects in Mice Lacking the Notch Ligand Jagged1," Human Molecular Genetics, Vol. 8, No. 5, 1999, pp. 723-730.

http://dx.doi.org/10.1093/hmg/8.5.723

[7] S.-M. Kwon, M. Eguchi, M. Wada, et al., "Specific Jagged-1 Signal from Bone Marrow Microenvironment Is Required for Endothelial Progenitor Cell Development for Neovascularization," Circulation, Vol. 118, No. 2, 2008, pp. 157-165.

http://dx.doi.org/10.1161/CIRCULATIONAHA.107.7549 $\underline{78}$

[8] X. Tong, Z. Yang, S. Xian, et al., "Clinical Observation on Mobilization Effect of Bone Marrow Stem Cells with Bushen Huoxue Fonmula in Acute Myocardial Infraction," Liaoning Journal of Traditional Chinese Medicine, Vol. 37, No. 10, 2010, pp. 1963-1965.

[9] X. Tong, Z. Yang, S. Xian, et al., "Study on Bushen Huoxue Recipe Mobilizing Marrow Stem Cells of Acute Myocadial Infraction in Rats," Chinese Journal of Basic Medicine in Traditional Chinese Medicine, Vol. 14, No. 8, 2008, pp. 588-591.

[10] D.-Q. Li, Y.-L. Duan, Y.-M. Bao, et al., "Neuroprotection of Catalpol in Transient Global Ischemia in Gerbils," Neuroscience Research, Vol. 50, No. 2, 2004, pp. 169177. http://dx.doi.org/10.1016/j.neures.2004.06.009 
[11] J. Seng, S. Luo, K. Ma, et al., "Research Advancements for Biomarkers Used in Acute Myocardial Infraction," Advices in Cardiovascular Diseases, Vol. 33, No. 1, 2012, pp. 106-106.

[12] L. Han and K. Chen, "Injurious Effect of Ischemic Reperfution and the Protective Effect of Yi-Xin-Kang Capsule on Rat Myocardial Mitochondria," Chinese Journal of Geriatric Cardiovascular and Cerebrovascular Diseases, Vol. 3, No. 5, 2001, pp. 331-332.

[13] E. Mannarino and M. Pirro, "Endothelial Injury and Repair: A Novel Theory for Atherosclerosis," Angiology, Vol. 59, No. 2, 2008, pp. 69S-72S. http://dx.doi.org/10.1177/0003319708320761

[14] C. Real, F. Caiado and S. Dias, "Endothelial Progenitors in Vascular Repair and Angiogenesis: How Many Are Needed and What to Do?" Cardiovascular \& Haemato- logical Disorders-Drug Targets, Vol. 8, No. 3, 2008, pp. 185-192.

[15] J. Lin, L. Xv, D. Yang, et al., "Effect of Tetramethylpyrazine on the Function of Endothelial Progenitor Cells in Vitro," Journal of Zhejiang University of Traditional Chinese Medicine, Vol. 32, No. 5, 2008, pp. 598-600.

[16] E. A. Jones, F. le Noble and A. Eichmann, "What Determines Blood Vessel Structure? Genetic Prespecification vs. Hemodynamics," Physiology, Vol. 21, No. 6, 2006, pp. 388-395. http://dx.doi.org/10.1152/physiol.00020.2006

[17] F. N. Karanu, B. Murdoch, L. Gallacher, et al., "The Notch Ligand Jagged-1 Represents a Novel Growth Factor of Human Hematopoietic Stem Cells," The Journal of Experimental Medicine, Vol. 192, No. 9, 2000, pp. 13651372. http://dx.doi.org/10.1084/jem.192.9.1365 